\title{
Forecasting electron cyclotron current drive stabilization of neoclassical tearing modes in ITER
}

Robert J. La Haye ${ }^{1}$ for the International Tokamak Physics Activity (ITPA) MHD, Disruptions and Control Topical Group Joint Experiment on Current Drive Prevention/Stabilization of Neoclassical Tearing Modes (MDC-8)

${ }^{1}$ General Atomics San Diego 92186 California USA

\begin{abstract}
The ITER baseline design relies on ECCD to stabilize confinementdegrading disruptive NTMs [1]. However, the EC power required will take a toll on the fusion gain $Q$. The MDC-8 group (in existence since 2005) has the goal to provide a range of data to benchmark the Rutherford tearing stability equation for NTM evolution, allowing predictions for ITER ECCD requirements to be validated. Experimental contributors have included ASDEX UPGRADE, DIII-D, EAST, FTU, HL-2A, JT-60U, KSTAR, TEXTOR and TCV. While any $\mathrm{m} / \mathrm{n}$ tearing mode island can reduce confinement, the $m=2, n=1$ mode at $q=2$ is particularly damaging. This mode is at a relatively large minor radius in the low q95 3 safety factor of ITER and thus close to the resistive wall; with the relatively low rotation in ITER (large inertia, small torque), an uncontrolled mode will rapidly lock at low tearing mode amplitude with subsequent disruption [2]. While progress is being made in modeling of the stability space and control [3] and experiments are promising, implementation still needs to be successfully demonstrated experimentally.

The ITPA consensus is that ITER's 24 1-MW gyrotrons will provide more than sufficient EC power from the upper launch mirrors to drive narrow (but not too narrow) $E C C D$ at $q=2$ for stabilization, with good alignment. Broadening of the ECCD, by edge turbulence for example, is a concern that would demand more EC power but also make alignment easier. Pre-emption at lower CW power or active stabilization by early mode onset detection and higher peak (possibly lower average) pulsed power are issues still under continuing investigation.

Most EC-NTM experimental studies so far are at relatively high q95 with smaller radius at $\mathrm{q}=2$ and thus higher Te for better current drive efficiency, higher rotation and weaker wall coupling. DIII-D, for example, is now well poised to pursue ECCD NTM stabilization at both low q95 and at low rotation in the 2017 campaign. The MDC-8 as a whole is proceeding to narrow the experimental focus for a comparison of observations from different devices. This will establish the physics basis for successful stabilization in ITER.
\end{abstract}

\section{Neoclassical tearing modes and their stabilization by ECCD}

NTMs are classically stable and neoclassically metastable requiring a seeding event to destabilize the mode [4]. The destabilizing helically perturbed neoclassical bootstrap current is counteracted at small island widths less than that of the ion banana width $\mathrm{w}_{\mathrm{ib}}$; everything else equal the NTM scaling is unfavorable for ITER as with about the same $\mathrm{w}_{\mathrm{ib}}$, the ratio of $\mathrm{w}_{\mathrm{ib}}$ to the minor radius $r_{s}$ at a rational surface is much smaller. This makes for a relatively easier to seed mode with a more virulent growth rate than in existing tokamaks. Well-aligned ECCD in the direction co to the plasma current direction can replace the missing bootstrap current stabilizing the mode, i.e. removing the metastable space. A simplified equation for an NTM is given as Eqn (1) of the modified Rutherford equation for normalized growth versus normalized island size. Key terms in Eqn (1) are given in [4]. Here $\mathrm{K}_{1}$ is an effectiveness parameter of order 1 for well-aligned ECCD that has width (" $\mathrm{w}_{\mathrm{cd}}$ " for

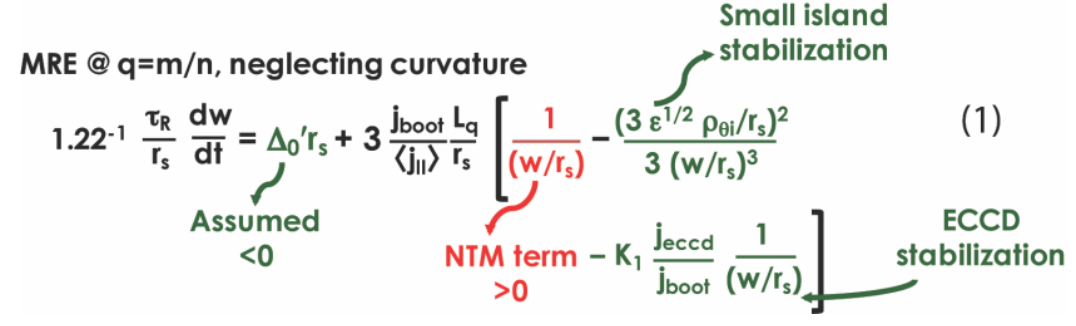




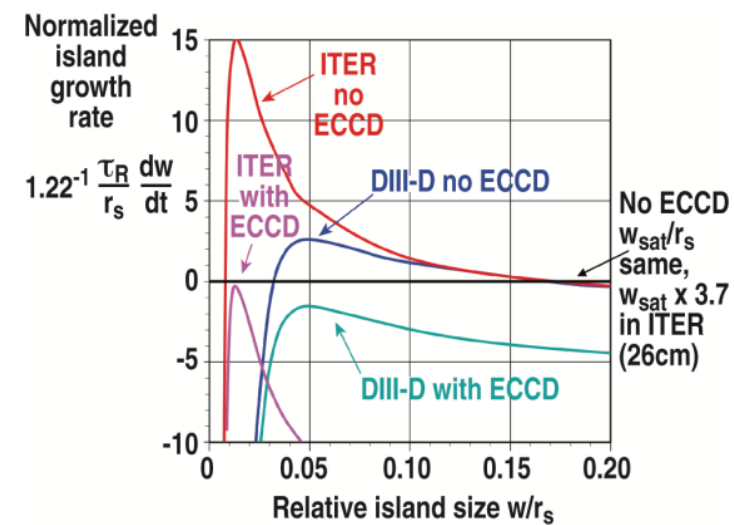

Fig 1. Normalized growth rates for DIII-D and ITER w/o ECCD and with well-aligned and matched by width ECCD of $\mathrm{j}_{\text {eccd }} / \mathrm{j}_{\text {boot }}=1.2$ for $\mathrm{K}_{1}=0.5$. ITER is $3.7 \mathrm{X}$ larger than DIII-D and all other quantities are same.

$\pm 1 / \mathrm{e})$ comparable to the most virulent largest growth rate island width (" $\mathrm{w}_{\text {marg }}$ ") w/o ECCD as shown in Figure 1. We will focus on the $m / n=2 / 1$ mode closest to the vessel wall that is the most damaging. The ITER upper launcher has good capability for NTM stabilization [5]. About 6 MW can drive the figure of merit $\mathrm{j}_{\text {eccd }} / \mathrm{j}_{\text {boot }}=1.2$ with a good match of $\mathrm{w}_{\mathrm{cd}}$ to the expected $\mathrm{w}_{\text {marg }}$ of 3 times the ion banana width for $\mathrm{q}=2$. Modeling is discussed more in 2 and experiments in 3.

\section{Power requirements for controlling NTMs by ECCD in ITER}

The fusion gain (Q) is improved by controlling NTMs by ECCD, and large islands that slow down, lock to the first resistive wall and induce disruption, are avoided, but the EC power required takes a toll on $\mathrm{Q}$. This is predicted in Figure 2 [6]. A belt model is used to estimate how the islands degrade the confinement for fixed heating power as compared to stabilization with ECCD power added. Modeling of co-ECCD stabilizes both by replacing the missing bootstrap current and/or by making classical tearing more

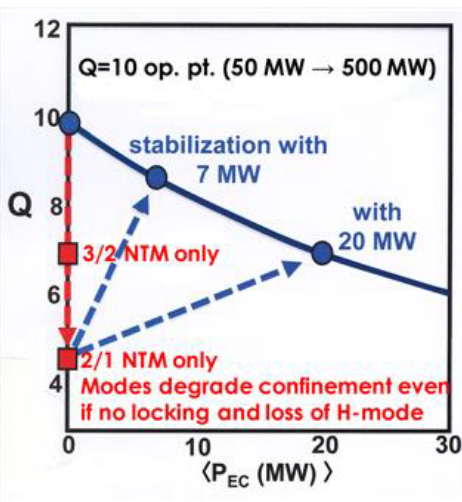

[Adapted from Sauter, EPS 2005 and Zohm, IAEA 2006]

Fig.2. Operating point Q for fusion gain in ITER with $3 / 2$ or $2 / 1$ islands (no EC) and islands stabilized (with EC).

stable. Studies of the minimum EC power requirement for the $2 / 1$ tearing mode in ITER make different physics assumptions but are consistent in the power requirements for ITER $[7-12,3]$. This is shown in Table 1. Control should minimize average EC power for successful stabilization subject to available peak EC power in ITER. Accurate real-time location of both $\mathrm{q}=2$ (MHD reconstruction EFIT with motional Stark effect pitch angle profile for example) and ECCD location (by TORBEAM) [13] could keep radial misalignment to no more than $\pm 1 \mathrm{~cm}$ comparable to DIII-D but relatively 4X more accurate in ITER. Control strategies to keep the EC power on at a minimum or off until needed are given in Figure 3. Pre-emption may require less peak power by avoiding a mode being destabilized, while standby has to be turned on when a mode is detectable and may already be larger width than that of the ECCD so that $\mathrm{K}_{1}$ is reduced [3]. However, catching a mode at low amplitude may be possible in ITER [14]. NTMs need seeding to be destabilized and seeding comes from predictably periodic MHD events such as $\mathrm{q}=1$ sawteeth crashes, $1 / 1$ fast

Table 1. Minimum CW EC power modeled needed to stabilize the $2 / 1$ NTM in ITER with different assumptions for $\mathrm{w}_{\mathrm{cd}}$ and $\mathrm{w}_{\mathrm{marg}}$ as well as classical tearing (not shown). Based on the MRE as given in Eqn (1).

\begin{tabular}{|l|c|c|l|}
\hline Reference & $\frac{\mathrm{W}_{\mathrm{cd}}(\mathrm{cm})}{2.5 \rightarrow 5}$ & 4 of $2,4,6$ scan & $\frac{\mathrm{w}_{\text {marg }}(\mathrm{cm})}{7 \rightarrow 9}$ \\
\hline Urso 2010 & 3.0 & 3.7 & 10 starting from full $\mathrm{w}_{\text {sat }}$ \\
\hline Bertelli 2011 & 5.0 & 4 of $2 \rightarrow 6 \mathrm{scan})$ & 9 \\
\hline van den Brand 2012 & 2.4 & 2 or 6 & 13 fixed for "latency" at $10 \mathrm{~cm}$ \\
\hline E. Poli 2015 & $2.8-3.7$ & 4 of $1 \rightarrow 6 \mathrm{scan}$ & 6 \\
\hline La Haye 2015 & 3.1 & 3.5 & $5 \sim 6$ \\
\hline F. Poli 2016 & 4 & 4.3 & $5 \sim 7$ \\
\hline
\end{tabular}




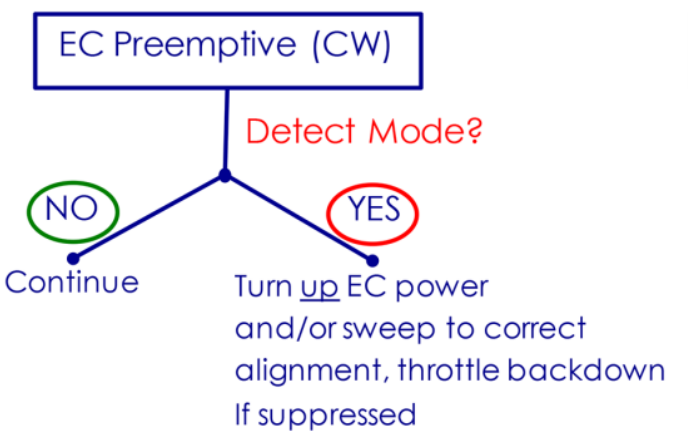



Fig. 3. Pre-emptive ECCD is CW with a minimum EC power with mirrors moving to stalk the $\mathrm{q}=2$ surface. Standby is mirrors moving to stalk $\mathrm{q}=2$ without $\mathrm{EC}$ power on until a mode detected and $\mathrm{EC}$ then turned on.

particle events known as fishbones or broad intermediate $n$ edge localized modes (ELMs). A standby control that is off most of the time with a higher peak power when on might have lower average EC power than that of a pre-emptive control that is always on. This is being tested experimentally as described next.

\section{Ongoing experimental studies of ECCD NTM stabilization and means of control}

Much experimental progress has been made since the MDC-8 group was founded in 2005. More gyrotrons for more power and more sophisticated means of controlling the EC power have been successfully implemented. Experiments are just getting underway on EAST and KSTAR and will be a key feature of JT60SA and WEST. Other experiments that have established and are active in establishing the physics basis were performed on ASDEX Upgrade, DIII-D, FTU, HL-2A, JT-60U, TCV and TEXTOR. Only a very few highlights can be noted here. Pre-emption requires good alignment of the ECCD on $\mathrm{q}=2$ rather than on an observable island. On TCV the mirrors were commanded to make multiple ECCD sweeps



Fig. 4. Pre-emptive ECCD stabilization of the $2 / 1$ tearing mode by mirror sweeping in TCV followed by the same applied to the pre-existing mode [15]. Same sweeping deposition whenever $\mathrm{EC}$ the power is on. back and forth across the radial position where $\mathrm{q}=2$ is expected to be; the mode did not appear until the EC power was turned off and when reapplied the mode was influenced but not stabilized [15]. This is shown in Figure 4 noting that EC is also used for core heating.

In the presence of a mode, here $\mathrm{m} / \mathrm{n}=3 / 2$, ASDEX Upgrade has used mirror sweeping over the island location which with enough EC power can be transiently good enough for stabilization [16]. This is shown in Figure 5. The mode amplitude is periodically reduced as the alignment is good but is only stabilized when the EC power is turned up.

On DIII-D pre-emption of the $2 / 1$ tearing mode in an ITER baseline scenario at normalized beta of $\beta_{N}=1.8$, safety factor at the $95 \%$ flux surface $\mathrm{q} 95=3.1$ and an equivalent low torque for low rotation and thus little if any tearing stabilization from flow shear is being studied with "Active Tracking". Real-time Thomson scattering for $\mathrm{n}_{\mathrm{e}}$ and $T_{e}$ profiles is used to aim the mirrors for ECCD at a $\mathrm{q}=2$ surface determined by real-time equilibrium reconstructions including the motional Stark effect magnetic pitch angle profile diagnostic. An offset can be programmed

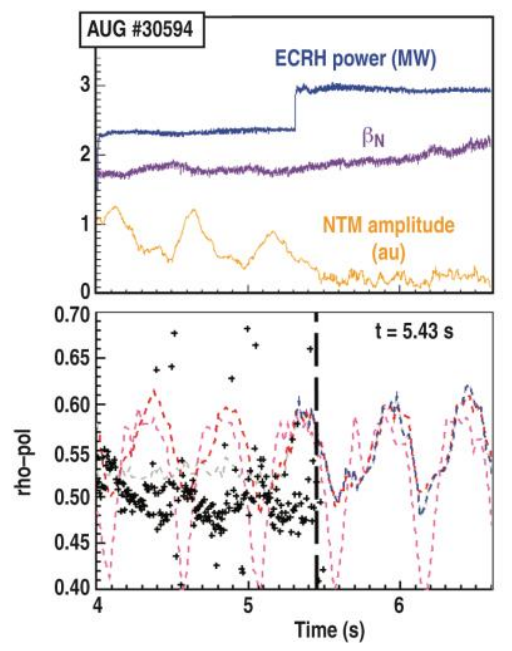

Fig. 5. ECCD stabilization of a $3 / 2$ tearing mode in Asdex Upgrade with mirror sweeping so that the alignment is transiently good enough for complete suppression with sufficient EC power [16]. Lower pane black crosses island location from ECE, dashes ECCD locations from each gyrotron when powered. 


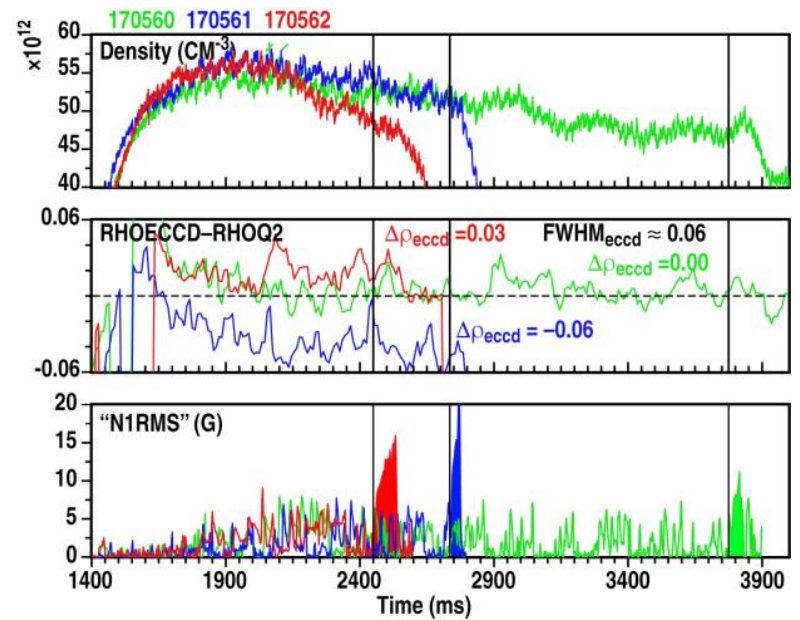

Fig. 6. Active tracking of ECCD on $\mathrm{q}=2$ in DIII-D with PCS keeping $\Delta \rho_{\text {eccd }}=0.00,0.03$ or $\mathbf{- 0 . 0 6}$ alignment.

into the plasma control system (PCS) for physics study of alignment or to correct a suspected systematic error. This is shown in Figure 6 for a very recent experiment. EC from 4 gyrotrons is applied at $\mathrm{t}=1400 \mathrm{~ms}$ just before the $\mathrm{L}$ to $\mathrm{H}$ transition that brings up the beta and density to flat top at $\sim 2000 \mathrm{~ms}$; this is followed by a natural very slow density decay during which the ECCD refraction is reduced while $\mathrm{q}=2$ remains the same, thus necessitating mirrors moving. While all 3 discharges eventually go unstable with the mode promptly locking, the longest delay is for best alignment; here power from 4 gyrotrons directed at $\mathrm{q}=2\left(2.4 \mathrm{MW}, \mathrm{j}_{\text {eccd }} / \mathrm{j}_{\text {boot }} \approx 0.8\right)$, is not enough while $5\left(2.8 \mathrm{MW}, \mathrm{j}_{\text {eccd }} / \mathrm{j}_{\text {boot }} \approx 1.0\right)$ can keep the $2 / 1$ mode from occurring as shown in Figure 7 . Here 0,4 or 5 gyrotrons are applied with different preset offsets using either "Active Tracking" of mirrors or "Point \& Shoot" fixed mirrors. The soon to be added new higher power gyrotron will allow better resolution of the stable/unstable space for $\mathrm{x}>0$ and $\mathrm{y}>1$, which is clearly not now warranted in Figure 7. Taken together the minimum in the figure of merit for successful pre-emption is about $\mathrm{j}_{\text {eccd }} / j_{\text {boot }} \sim 1$. This requires the EC power to be about $50 \%$ of the total input power including that of neutral beam injection.

*This work is supported in part by the US DOE under DE-FC02-04ER54698 and the DIII-D 3D Stability Physics and EC Operations Groups are acknowledged. The ITPA MDC-8 participants include M. Reich and E. Westerhof for ASDEX Upgrade, R. La Haye for DIII-D, L. Hu for EAST, S. Novak for FTU, Y. Liu for HL-2A, P. Buratti for JET, A. Isayama for JT-60SA, M. Joung for KSTAR, S. Sabbagh for NSTX-U, M. Reich and O. Sauter for TCV and P. Maget for WEST.

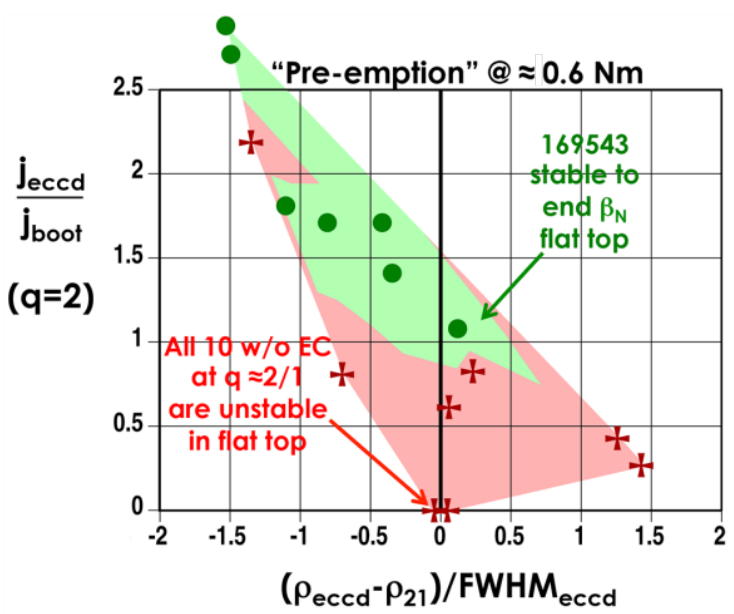

Fig. 7. Pre-emption by ECCD of $2 / 1$ tearing mode in DIII-D low torque IBS. Red crosses are at onset of mode. Green dots are no mode. Contours are pink mode unstable and green mode stable

\section{References}

[1] T.C. Hender, et al., Nuclear Fusion 47, S128 (2007)

[2] R.J. La Haye, C. Paz-Soldan and Y.Q. Liu, Nuclear Fusion 57, 014004 (2017)

[3] F. M. Poli et al., 26th IAEA Fusion Energy Conf, Kyoto, P4-6 (2016)

[4] R.J. La Haye, Phys. Plasmas 13, 055501 (2006)

[5] L. Figini et al., Plasma Phys. Control. Fusion 57, 054015 (2015)

[6] O. Sauter, H. Zohm, 32nd EPS Conference on Plasma Phys. Tarragona, 27 June - 1 July 2005 ECA Vol.29C, P-2.059 (2005)

[7] O. Sauter, et al., Plasma Phys. Control Fusion 52, 025002 (2010)

[8] L. Urso et al., Nucl. Fusion 50, 025010 (2010)

[9] N. Bertelli, et al., Nucl. Fusion 51, 103007 (2011)

[10] H. van den Brand et al., Plasma Phys. Control. Fusion 54, 094003 (2012)

[11] E. Poli, et al, Nuclear Fusion 55, 013023(2015)

[12] R. La Haye for the DIII-D Electron Cyclotron Operations and Dynamics \& Control Groups, $21^{\text {st }}$ rfPP Conference, Lake Arrowhead, 27-29 April, Volume 1689, 030018 (2015)

[13] E. Kolemen, et al., Nucl. Fusion 54, 073020 (2014)

[14] K E J Olofsson, et al., Plasma Phys. Control. Fusion 56, 095012 (2014)

[15] D. Kim, PhD thesis 2015 EPFL https://infoscience.epfl.ch/record/205040/files/E PFL TH6539.pdf

[16] M. Reich, et al., Fusion Science and Technology 61, 309 (2012) 